\title{
From local to global additive representation
}

\author{
Alain Chateauneuf \\ University of Paris I, CERMSEM, Paris, France \\ Peter Wakker* \\ University of Nijmegen (NICI), Nijmegen, The Netherlands \\ Submitted October 1991, accepted September 1992
}

This paper studies continuous additive representations of transitive preferences on connected subdomains of product sets. Contrary to what has sometimes been thought, local additive representability does not imply global additive representability. It is shown that the result can nevertheless be established under some additional connectedness conditions. This generalizes previous results on additive representations on (subsets of) product sets.

\section{Introduction}

The modern interest in additive representations on subsets of product sets stems from developments in decision making under risk/uncertainty, and welfare theory. There one wishes to deviate from the classical expected utility/utilitarianism paradigm [see Fishburn (1988) and Chew and Epstein (1989a), respectively] by weakening the independence axiom and requiring it to hold only on certain subsets of the space. Already Krantz, Luce, Suppes and Tversky (1971), hereafter abbreviated KLST, pointed out the importance of additive representations on subsets of product sets (see section 6.13 there). Fishburn (1976) studied additive representations on subsets of product sets, linear with respect to a mixture operation.

Necessary and sufficient conditions for general additive representation on arbitrary countable subsets of product sets were provided by Jaffray (1974b) for two dimensions, and Jaffray (1974a) for arbitrary finite dimensions. Fishburn (1992) extended these results and showed their relevance for many

Correspondence to: Peter Wakker, University of Leiden (AZL), Medical Decision Making Unit (Bldng 43), P.O. Box 9600, Leiden, 2300 RC, The Netherlands.

*The research of Peter Wakker has been made possible by a fellowship of the Royal Netherlands Academy of Arts and Sciences, and a fellowship of the Netherlands Organization for Scientific Research. 
related problems. We study the special case of additive representations that are continuous with respect to a connected topology, without any assumption of linearity. For this case, a first approach was given in Wakker (1986 chapter VI, 1989a, 1989b - chapter VI), where implicitly additive representation results were derived on 'rank-ordered sets' (defined in Assumption C.2) in order to derive Choquet expected utility. Choquet expected utility, introduced by Schmeidler (1989), is one of the recent nonexpected utility models.

Green and Jullien (1988) used the same technique to generalize rankdependent utility as introduced by Quiggin (1982), and Chew and Epstein (1989b) used it for further generalizations. Problems for these approaches were pointed out by Wakker, for instance in Wakker (1993). The latter paper explicitly derived results for rank-ordered sets within connected topological spaces. Primarily to help correcting the results of Chew and Epstein (1989b), Segal (1991) gave results for connected domains which satisfy some additional connectedness conditions [(2) and (3) below], for the special case of open subsets of Euclidean spaces; for this case his domains are considerably more general than rank-ordered sets. Segal dealt with the case of three or more dimensions. When his additional connectedness conditions are adapted to the, simpler, case of two dimensions, then already Blaschke and Bol (1938) obtained such results for domains that are simply connected (which is less general than connected). Segal also gave some results for closed sets.

This paper uses the same additional connectedness conditions, and generalizes Segal's results as follows:

- First, Segal's results for nonopen domains are generalized. All problems that may occur on nonopen domains are identified.

- Second, the domain is generalized to connected topological spaces. This includes multi-dimensional commodity bundles, and nonquantified outcomes. Further, also the case of two dimensions is enclosed, generalizing Blaschke and Bol's (1938) results to connected instead of simply connected domains.

- Third, it is observed that the characterizing conditions need only be required locally. Indeed, this paper first shows how local additive representability implies global additive representability. Next representation results are obtained by establishing local additive representability. For full product sets, Wakker (1990) showed that local additive representability implies global additive representability; Chateauneuf subsequently found a simpler proof. See also Vind (1987 - Corollary IV.2.6, 1991 - p. 134). He obtained that result when local additive representability of $\geqslant$ can be extended to a local mean groupoid operation on the set of equivalence classes. Note that some uniformity or restriction to compact domains is needed for that extension.

Lemmas C.1 and C.3 show that full product sets, as well as rank-ordered sets, satisfy all connectedness conditions required for our results. Note also 
that the discussion of nonopen domains in section 3 includes the nonopen rank-ordered sets. Thus the present paper provides a joint generalization of Wakker (1993) and Segal (1991). Proofs will be simplified as compared to these references. Increased generality of results often concurs with increased simplicity of proof; we hope that the present paper can serve as an illustration.

\section{Elementary definitions, and results for open domains}

Let, for $n \geqq 2, X_{1}, \ldots, X_{n}$ be nonempty sets; alternatives are elements of $\prod_{i=1}^{n} X_{i}$, denoted as $x=\left(x_{1}, \ldots, x_{n}\right)$, etc. By $y_{i} x$ we denote the alternative $(x$ with $x_{i}$ replaced by $y_{i}$ ). Let $\geqslant$ be a weak order on a subset $E$ of $\prod_{i=1}^{n} X_{i}$, i.e., a binary relation that is transitive and complete (thus reflexive). As usual, $>$ denotes the asymmetric part, $\sim$ the symmetric part, and $\preccurlyeq$ and $\prec$ denote reversed binary relations. We write $] x, z[$ for $\{y \in E: x \prec y \prec z\}$, $] x, \rightarrow[$ for $\{y \in E: x \prec y\},] \leftarrow, z[$ for $\{y \in E: y \prec z\}$, and $[x, z]$ for $\{y \in E: x \preccurlyeq y \preccurlyeq z\}$. Similar notations are $[x, z[$ and $] x, z]$ and $[x, \rightarrow[$ and $] \leftarrow, z]$. Note that $\sim$ is an equivalence relation, with sets $[x, x]$, or $[x]$ for short, as equivalence classes.

We assume that on each $X_{i}$ a weak order $\succcurlyeq_{i}$ is given. For the $\succcurlyeq_{i}$ relations

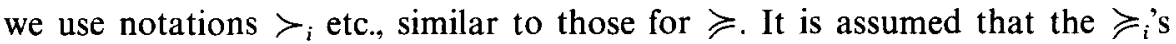
are nontrivial, i.e., $x_{i} \succ_{i} y_{i}$ for some $x_{i}, y_{i}$. Further $\geqslant$ is assumed to satisfy (strong) monotonicity, i.e.,

$$
\left[\forall i: x_{i} \succcurlyeq_{i} y_{i}\right] \Rightarrow x \geqslant y \text { and }\left(\left[\forall i: x_{i} \succcurlyeq_{i} y_{i}\right] \text { and }\left[\exists i: x_{i} \succ_{i} y_{i}\right]\right) \Rightarrow x>y \text {. }
$$

Each $X_{i}$ is endowed with the order topology of $\geqslant_{i}$, i.e., the smallest topology containing all sets of the form $] x_{i}, \rightarrow[$ and $] \leftarrow, z_{i}[$, thus also sets of the form ]$x_{i}, z_{i}\left[. \prod_{i=1}^{n} X_{i}\right.$ is endowed with the product topology, $E$ with the restriction thereof; the binary relation $\geqslant$ is assumed continuous, i.e., for each $x \in E$, $\{y \in E: y \geqslant x\}$ and $\{y \in E: y \preccurlyeq x\}$ are closed within $E$. As usual, int denotes topological interior, and cl denotes topological closure.

Cubes are subsets that are products of 'order intervals' $] x_{i}, z_{i}[$ or $] x_{i}, \rightarrow[$ or $\ldots$ or $\left.] \leftarrow, z_{i}\right]$. A function $V: E \rightarrow \mathbb{R}$ (globally) represents $\geqslant$ if $[\forall x, y \in E$ : $x \geqslant y \Leftrightarrow V(x) \geqq V(y)]$. It is additive if there exist 'coordinate functions' $\left(V_{j}\right)_{j=1}^{n}$ containing the projections of $E$ on $X_{j}$ in their domain, and such that $V(x)=\sum_{j=1}^{n} V_{j}\left(x_{j}\right)$ for all $x$. If $V$ is additive and represents $\geqslant$, then the $V_{j}$ 's are called additive value functions. We say that $\geqslant$ is locally additively representable if for each element in the domain of $\geqslant$ there exists an open neighborhood on which there exists an additive representation. A function is rescaled if it is multiplied with a positive real number, and a real number is added to it. An additive representation is usually cardinal, i.e., another function is an additive representation if and only if it can be obtained by rescaling the first function. 
For easy reference, let us display the assumptions that we use in the main results:

Assumption 2.1 (Structural Assumption). $\left(X_{i}\right)_{i=1}^{n}$ are nonempty sets endowed with nontrivial weak orders $\succcurlyeq_{i}$ and their order topologies; $n \geqq 2$. $\prod_{i=1}^{n} X_{i}$ is endowed with the product topology. A monotonic continuous weak order $\succcurlyeq$ is given on a set $E \subset \prod_{i=1}^{n} X_{i}$. The following sets are connected:

$$
\begin{aligned}
& \operatorname{int}(E) \text {; } \\
& \text { all sets of the form: }\left\{x \in \operatorname{int}(E): x_{i}=s_{i}\right\} \text { for some } i, s_{i} \text {; } \\
& \text { all } \sim \text { equivalence classes } \operatorname{in} \operatorname{int}(E) .
\end{aligned}
$$

Next we give the representation theorem on open domains. It will be proved in Appendix A.

Theorem 2.2. Assume the Structural Assumption 2.1, and suppose E is open. Then local additive representability implies global additive representability. The gobal additive representation is continuous and cardinal.

The idea of the proof is to start with the additive representation on one open cube, and then add, one by one, new cubes. On the overlap with the area already covered, the local additive representation on a new cube can be fitted together with the function as already constructed; thus an extension of domain has been obtained. The process stops when the entire domain has been covered. As pointed out already in KLST (subsection 6.5.5), in this reasoning several consistencies must be established. Condition (1) above guarantees that indeed the process will only stop if the entire domain has been covered (see Stage 3 in the proof in Appendix A). Condition (2) implies, for any $i$, that from different (remote) local domains never contradictory requirements result for the additive value function $V_{i}$ (see Step 2.2 in the proof). Thus the function as constructed remains additively decomposable. This uses the idea of Lemma 1 in Segal (1991) (adapted to our more general context by Lemma B.3). Condition (3) guarantees that the function as constructed is constant on $\sim$ equivalence classes; it then readily follows that the function is representing; see Stage 4 in the proof.

In the literature, conditions for preference relations $\geqslant$ have been studied that imply, in the presence of other conditions, additive representability on full product sets. Before presenting them, let us mention that in definitions below we omit quantifiers; these should be: for all alternatives in the domain. The most well-known condition for preference relations, mainly useful for 
$n \geqq 3$, is independence: $v_{i} x \geqslant v_{i} y \Leftrightarrow w_{i} x \geqslant w_{i} y$. For the case $n=2$, other conditions must be used, such as the Thomsen condition: $\left[\left(y_{1}, a_{2}\right) \sim\left(x_{1}, b_{2}\right) \&\left(x_{1}, c_{2}\right) \sim\left(v_{1}, a_{2}\right) \Rightarrow\left(y_{1}, c_{2}\right) \sim\left(v_{1}, b_{2}\right) \quad\right.$ [or 'triple cancellation', or the 'hexagon condition', see KLST, or Wakker (1989b)]. These conditions can be weakened, e.g., by the famous result of Gorman (1968). We shall summarize conditions as above as 'additivity axioms'. So we say the additivity axioms are satisfied if :

for $n=2$, the Thomsen condition holds, or triple cancellation, or the hexagon condition,

and

for $n \geqq 3$, independence is satisfied, or one of the weakenings of independence, derivable from Gorman (1968), that still imply independence on full product sets.

These conditions, in the presence of the other conditions of Theorem 2.2, straightforwardly imply local additive representability: Each alternative is contained in an open cube within the domain. This cube is a connected [see Lemma B.6(c)] full product set, with all coordinates essential by nontriviality of the $\succcurlyeq_{i}$ relations and monotonicity. So on this cube the additivity axioms imply additive representability by standard results, see for instance Debreu (1960), Gorman (1968), KLST, or Wakker (1989b). This gives the most important implication of Theorem 2.2:

Corollary 2.3. Assume the Structural Assumption 2.1, and suppose E is open. Then the additivity axioms for $\geqslant$ imply additive representability. The global additive representation is continuous and cardinal. The additivity axioms can be weakened to hold only locally.

Segal (1991) pointed out that the above connectedness conditions are mutually independent. They may be taken as formalization of 'something rcsembling a convex region' as it was formulated by KLST in subsection 6.5.5. Indeed, on a convex region conditions (1) and (2) are immediately satisfied; condition (3) may still be violated, and examples can show that the above results need not hold on convex domains. Since the problem is essentially topological, no central role should be expected for linear-space structures such as convexity.

Note that we can always restrict attention to the case where, for each $i, X_{i}$ is the projection of $E$. Then, if an additive representation exists, we take for the $\geqslant_{i}$ relations the relations represented by the additive value functions, and thus have monotonicity always satisfied. This shows that our assumption about the presence of the $\vartheta_{i}$ relations, and monotonicity with respect to these, is not a restriction in a structural sense: it is necessary for additive 

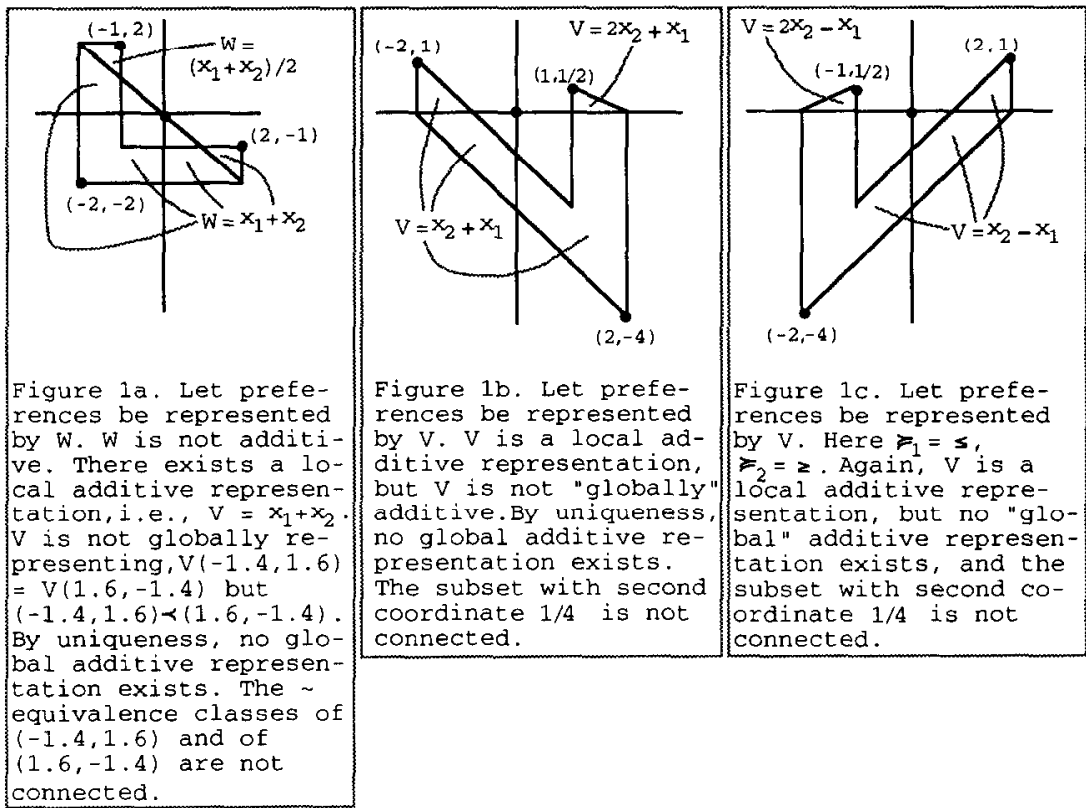

Fig. 1. [Necessity of Conditions (2) and (3)]. In all figures, the domain is the interior. Fig. 1c results from $1 \mathrm{a}$ by interchanging second coordinate and the representing function $(W$ or $V$ ), $1 \mathrm{~b}$ results from 1c by multiplying $x_{1}$ by -1 .

representability. That the connectedness conditions (2) and (3) cannot be omitted is shown in fig. 1. Comments and references concerning these examples are given in section 4 . If condition (3) were omitted, then $E$ could be partitioned into connected components. On each of these an additive representation can then be obtained. The separate components then do not have any equivalence class in common, and neither any coordinate, by (2) and (3); this excludes certain kinds of inconsistencies. There may, however, occur problems about 'driven to infinity' [see Segal (1991, Example 2)] or cardinality-in-the-set-theoretic-sense (more equivalence classes than real numbers). Also cardinality-in-the-measurement-uniqueness sense of this paper would change. For brevity we do not elaborate.

\section{Nonopen domains}

In this section we assume again that the Structural Assumption 2.1 holds. Note that the restriction of $\geqslant$ to $\operatorname{int}(E)$ is again continuous. We assume that, as in Theorem 2.2, an additive representation $V$ is given on int $(E)$. Extension of the additive representation from int $(E)$ to the boundary points of $E$ that are limits of interior points is straightforward by continuity, as we shall see. 


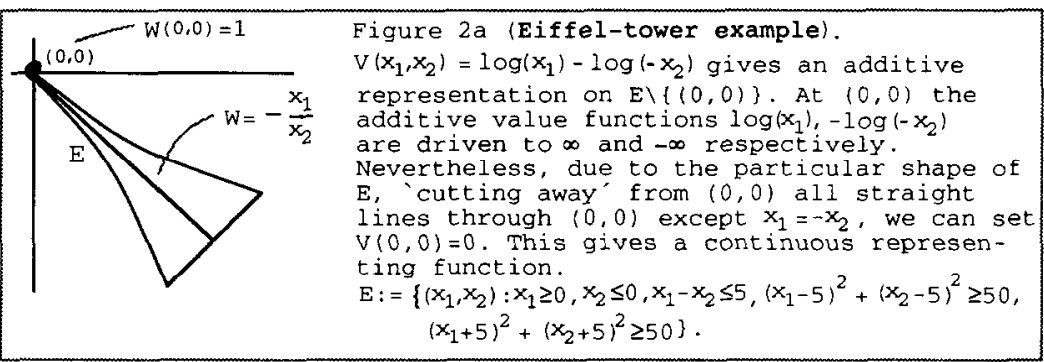

\begin{tabular}{|ll}
\hline$(1,1)$ & Figure $2 b$. \\
& $\left.V\left(x_{1}, x_{2}\right)=\log \left(x_{1}\right)+\log \left(x_{2}\right)\right)$ gives an additive \\
& representation on $E \backslash\{(0,0)\}$. At $(0,0)$ the \\
\hline$(0,0)$ & additive value functions $10 g\left(x_{1}\right), \log \left(x_{2}\right)$, as \\
& well as $v$, are driven to $-\infty$.
\end{tabular}

\begin{tabular}{l}
$\begin{array}{l}\text { wis a } \\
\text { monotonic } \\
\text { additive } \\
\text { function } \\
\text { with maxi- } \\
\text { mum at } x\end{array}$ \\
\hline$(0,0)$
\end{tabular}$\quad \begin{aligned} & x \text { is maximal but must have a finite value } \\
& \text { because it is interior-matched. }\end{aligned}$

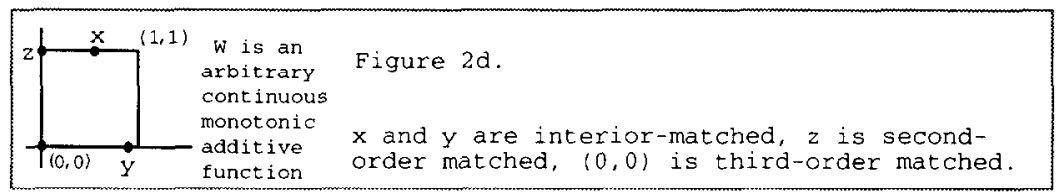

Fig. 2 (Driven to infinity). Preferences are represented by the function $W ; \bullet-$ points on the boundary are included in the domain $E$.

Only one problem can occur: the representing function, or some of the additive value functions, may be 'driven to infinity'. This was first shown by Wakker (1991, Example 25); see also Wakker (1993, Example 3.8). A small variation on this example is given in fig. $2 b$. The driven-to-infinity phenomenon is particularly problematic if it leads to summation of $\infty$ and $-\infty$; see the 'Eiffel-Tower' example in fig. 2a. ${ }^{1}$ For boundary points that are no limits of interior points, little can be said. Fig. 3 illustrates. Hence we assume:

All boundary alternatives are limits of interior alternatives.

We first show how to extend the definitions of the functions $V$ and $V_{1}, \ldots, V_{n}$ from int $(E)$ to $E$. The extended functions will be denoted by the same characters. They may take values $\infty$ or $-\infty$. Later we will establish continuity, and the additivity equation $V(x)=V_{1}\left(x_{1}\right)+\cdots+V_{n}\left(x_{n}\right)$. Let $\pi_{i}$ denote projection on the $i$ th coordinate. It is continuous, so assigns

\footnotetext{
${ }^{1}$ This example was actually found with a view on the Eiffel Tower, and the form of the domain was inferred from the form of the Eiffel Tower.
} 

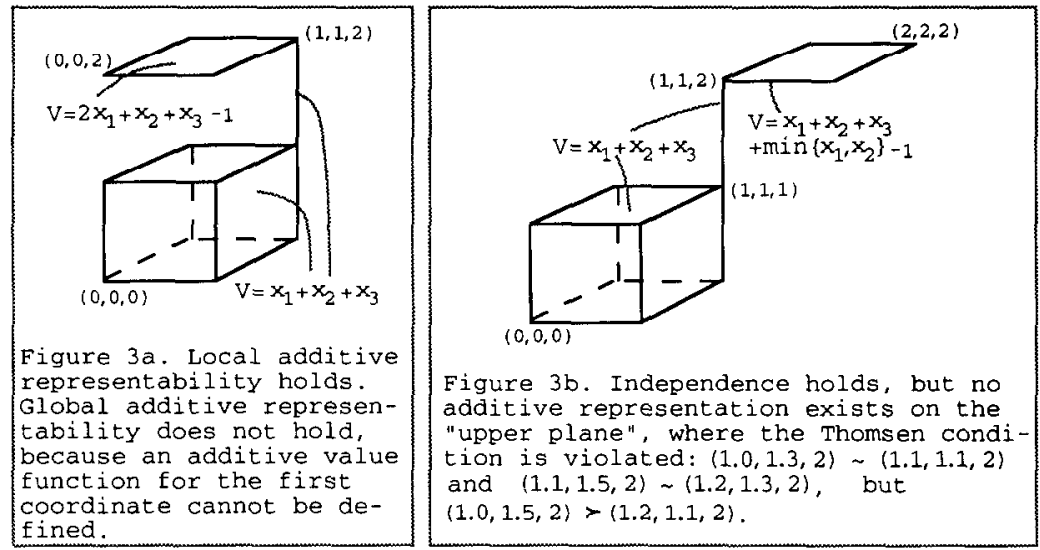

Fig. 3 (Boundary points that are no limits of interior points). Preferences are represented by the function $V$.

connected images to int $(E)$; also it is well-known that $\pi_{i}$ assigns open images to open sets, so $\pi_{i}($ int $(E))$ is open.

Lemma 3.1. If $x_{i} \notin \pi_{i}(\operatorname{int}(E))$, then $x_{i}$ is extreme, and either $x_{i} \succ_{i} y_{i}$ for all $y_{i} \in \pi_{i}(\operatorname{int}(E))$, or $x_{i} \prec_{i} y_{i}$ for all $y_{i} \in \pi_{i}(\operatorname{int}(E))$.

Proof. Suppose $x_{i}$ is not extreme, i.e., $v_{i} \prec_{i} x_{i} \prec_{i} y_{i}$ for some $v, y \in E$. Since $v$ and $y$ are limits of elements of int $(E)$, and topologies are generated by the relations $\succcurlyeq_{j}$, there are $v^{\prime}, y^{\prime} \in \operatorname{int}(E)$ with $v_{i}^{\prime}<_{i} x_{i} \prec_{i} y_{i}^{\prime}$. By connectedness and openness of $\pi_{i}(\operatorname{int}(E))$ and Lemma B.6(c), $x_{i} \in \pi_{i}(\operatorname{int}(E))$.

Also, if there were a $v_{i} \in \pi_{i}(\operatorname{int}(E))$ with $v_{i} \sim_{i} x_{i}$, then, because $\pi_{i}(\operatorname{int}(E))$ is open with respect to the order topology of $\succcurlyeq_{i}, x_{i} \in \pi_{i}(\operatorname{int}(E))$ would follow.

If $x_{i} \succ_{i} y_{i}$ for all $y_{i} \in \pi_{i}(\operatorname{int}(E))$ then we call $x_{i}$ maximal, if $x_{i} \prec_{i} y_{i}$ for all $y_{i} \in \pi_{i}\left(\operatorname{int}(E)\right.$ ) then we call $x_{i}$ minimal; these terms are relative to $\pi_{i}(E)$ (not necessarily to $\left.X_{i}\right)$. For maximal $x_{i}$ we define $V_{i}\left(x_{i}\right):=\sup \left(V_{i}\left(\pi_{i}(\operatorname{int}(E))\right)\right)$, for minimal $x_{i}, V_{i}\left(x_{i}\right):=\inf \left(V_{i}\left(\pi_{i}(\operatorname{int}(E))\right)\right)$.

\section{Lemma 3.2. On $\pi_{i}(E), V_{i}$ represents $\geqslant_{i}$ and is continuous.}

Proof. In the proof of Theorem 2.2, it is shown (in Step 4.3, and Stage 5, in Appendix A) that each $V_{i}$ is continuous and representing on $\pi_{i}(\operatorname{int}(E))$. We first show that $V_{i}$ is representing on the entire set $\pi_{i}(E)$.

If $x_{i}$ and $y_{i}$ are both maximal, then $x_{i} \succ_{i} y_{i}$ would contradict the extremity of $y_{i}$, obtained in Lemma 3.1. So $x_{i}$ and $y_{i}$ are equivalent; indeed they also have the same $V_{i}$ value. Next consider the case of a maximal $x_{i}$ and a 
nonmaximal, possibly minimal, $y_{i}$. We have $x_{i} \succ_{i} y_{i}$ so, by continuity, $x_{i} \succ_{i} v_{i} \succ_{i} w_{i} \succ_{i} y_{i}$ for some $v_{i}, w_{i} \in \pi_{i}(\operatorname{int}(E))$, giving $V_{i}\left(x_{i}\right) \geqq V_{i}\left(v_{i}\right)>V_{i}\left(w_{i}\right) \geqq V_{i}\left(y_{i}\right)$. A similar reasoning applies to the case of $x_{i}, y_{i}$ where $x_{i}$ is minimal. It follows that $V_{i}$ is representing.

Continuity of $V_{i}$ on $\pi_{i}(E)$ follows as the range of $V_{i}$ contains no gaps, and the represented $\succcurlyeq_{i}$ is continuous on the connected space $\pi_{i}(E)$. The latter connectedness follows because $\pi_{i}$ (int $(E)$ ) is connected and open, so is a preference interval by Lemma B.6(c). It still is a preference interval if the maximal and minimal coordinates are added, after which $\pi_{i}(E)$ results. By Lemma B.6(c), $\pi_{i}(E)$ is connected.

Next we turn to the extension of the function $V$. As this is similar to the extension of the functions $V_{i}$, we shall describe it more briefly. If $x \sim y$ for some $y \in \operatorname{int}(E)$, then we define $V(x):=V(y)$. If no such interior 'matching' point $y$ exists, then by connectedness and continuity either $x>y$ for all interior $y$ ( $x$ is maximal), or $x<y$ for all interior $y$ ( $x$ is minimal). In the former case we define $V(x):=\sup (V(\operatorname{int}(E)))$, in the latter case $V(x):=$ $\inf (V(\operatorname{int}(E)))$. It straightforwardly follows that $V$ represents $\succcurlyeq$ and is continuous (its range containing no gaps).

Now suppose that $V(x)$, as well as all $V_{j}\left(x_{j}\right)$ 's, are finite, and that $x$ is a limit of interior $x^{i}$ 's. ${ }^{2}$ Then

$$
V(x)=\lim _{i \rightarrow \infty} V\left(x^{i}\right)=\lim _{i \rightarrow \infty} \sum_{j=1}^{n} V_{j}\left(x_{j}^{i}\right) \stackrel{(1)}{=} \sum_{j=1}^{n} \lim _{i \rightarrow \infty} V_{j}\left(x_{j}^{i}\right)=\sum_{j=1}^{n} V_{j}\left(x_{i}\right)
$$

as desired.

Now we turn to the only problem for extendability to the boundary: Some of the $n+1$ values $V(x), V_{1}\left(x_{1}\right), \ldots, V_{n}\left(x_{n}\right)$ may be infinite. In particular, if in (4) some terms of the right-hand side of $\stackrel{(1)}{=}$ are $\infty$ and others are $-\infty$, the summation at the right-hand side of $\stackrel{(1)}{=}$ is not well defined. The reasoning in (4) also shows that never only one of $V(x), V_{1}\left(x_{1}\right), \ldots, V_{n}\left(x_{n}\right)$ can be infinite. So, if for an alternative $x$, at least $n$ of the values $V_{1}\left(x_{1}\right), \ldots, V_{n}\left(x_{n}\right), V(x)$ are finite, then in fact all $n+1$ of these values are finite. It follows inductively that all functions $V_{1}, \ldots, V_{n}, V$ are finite, so an additive representation is obtained, at boundary points of the following kind:

(1) The interior-matched alternatives $x$, i.e., of $[x], x_{1}, \ldots, x_{n}$, at most one does not occur for an interior alternative, so is maximal or minimal.

(2) The second-order matched alternatives $x$, i.e., of $[x], x_{1}, \ldots, x_{n}$, at most one does not occur for an interior, or interior-matched, alternative.

\footnotetext{
${ }^{2}$ We may, and will, assume that $x$ is the limit of a countable sequence $\left(x^{i}\right)_{i=1}^{\infty}$. This follows because, after the collapsing of $\sim_{1} \ldots \sim_{n}$ equivalence classes, the mapping $\left(x_{1}, \ldots, x_{n}\right) \mapsto$ $\left(V_{1}\left(x_{1}\right), \ldots, V_{n}\left(x_{n}\right)\right)$ is a homeomorphism from int $(E)$ to a subset of $\mathbb{R}^{n}$.
} 
(3) The third-order matched alternatives $x$, i.e., of $[x], x_{1}, \ldots, x_{n}$, at most one does not occur for an interior, interior-matched, or second-order matched alternative.

$(2 n+2)$. The $(2 n+2)$ th-order matched alternatives $x$, i.e., of $[x], x_{1}, \ldots, x_{n}$, at most one does not occur for an interior, interior-matched, second-order matched, $\ldots$, or $(2 n+1)$ th-order matched alternative.

We call an alternative matched if it is interior, interior-matched, or matched of any order. Interior-matched alternatives, second-order matched alternatives, and third-order matched alternatives, are illustrated in fig. $2 \mathrm{~d}$. Note that every new order of matched alternatives shows finiteness of at least one new maximal or minimal coordinate or $\sim$ equivalence class. Hence no more than $2 n+2$ orders will include new coordinates or alternatives.

Segal (1991) proposed (for compact domains with Euclidean spaces) the following condition: For each extreme coordinate $x_{i}$ there exist $y, z, w$ such that $y_{i}=z_{i}=w_{i}=x_{i}$, and for each $j \neq i, y_{j} \succ_{j} z_{j} \succ_{j} w_{j}$. This condition implies that $z$ is interior-matched, and that each alternative is second-order matched, so that Segal's result is implied by the results of this section. Wakker (1991, 1993), for rank-ordered sets, gave alternative conditions to rule out the driven-to-infinity problem. The conditions were strengthenings of the 'Archimedean axiom'. Their meaning is sensitive to the form of the domain, and we are not aware of an adaptation to more general domains such as considered in this paper.

We summarize the results obtained in this section:

Theorem 3.3. Assume the Structural Assumption 2.1. Suppose that int $(E) \subset$ $E \subset \operatorname{cl}(\operatorname{int}(E))$. Then:

(a) Local additive representability on int $(E)$ implies global additive representability on the set of matched alternatives, thus obviously on any of its subsets. The global additive representation is continuous and cardinal.

(b) The additivity axioms for $\geqslant$ on int $(E)$ imply additive representability on the set of matched alternatives (or any of its subsets). The global additive representation is continuous and cardinal. The additivity axioms can be weakened to hold only locally.

\section{Further remarks, examples, and a useful symmetry}

As demonstrated in Wakker (1993, Example 3.9), continuity of an additive representing function need not imply continuity of the additive value functions, if the domain is a general subset of a product set. By Lemma 3.2, all additive value functions in this paper are continuous and representing. 
Note that it is essential for our analysis that the topologies on the sets $X_{i}$ are generated by the orders $\succcurlyeq_{i}$. This is necessary because the open neighborhoods on which an additive representation is given should contain a connected cube of the form $\left.\prod_{i=1}^{n}\right] a_{i}, b_{i}[$. No topological separability has been assumed. Topological separability of the order topologies does hold, it is implied by representability, thus is implied by the other conditions in Theorem 3.3.

Topological connectedness and the absence of 'holes' 3 has played a central role in the proof. This suggests that a generalization to the algebraic set-up of KLST, with an Archimedean axiom and restricted solvability instead of continuity with respect to a connected topology, will not work. In such a generalization, neighborhoods would be replaced by cubes of the form I $\left.\int_{i=1}^{n}\right] x_{i}, y_{i}[$. We have neither a proof nor a counterexample to such a generalization. The generalization can of course be obtained by adding as additional assumptions the implications of topological connectedness that are used in the proof, i.e., the several usages of linkedness.

The reader will have noted that the conditions for the sets of the form $\left\{x \in E: x_{i}=s_{i}\right\}$, for some $i, s_{i}$, were the same as for the $\sim$ equivalence classes. Also it will be noted that the derivation of representability of $V$ resembled the derivation concerning consistency of $V_{1}, \ldots, V_{n}$. Same symmetries occurred at the extension to boundary alternatives. Indeed, there is an underlying symmetry between the $n+1$ binary relations $\geqslant_{1}, \ldots, \geqslant_{n}, \geqslant$, similarly between the subsets where some coordinate is fixed, and the equivalence classes.

A first understanding of this symmetry can be obtained from the way in which web theory results from Blaschke and Bol (1938) have been used in additive representation theory, for instance in Debreu (1960). To illustrate this, take $\mathbb{R}^{2}$ as domain. Web theory considers three families of curves in the plane, and transforms them into families of parallel curves. The role of these families is entirely symmetric. For application to additive representation theory, the curves of one of the three families are transformed into lines where the first coordinate is constant, the curves of a second family are transformed into lines with constant second coordinate, and, finally, the curves of the third family are the equivalence classes of the preference relation. This symmetry has been explicated in KLST (subsection 6.5.6).

The main result of this paper could have been reformulated as a result where in an $(n+1)$-dimensional product set $[$ the $(n+1)$ th dimension describing $\sim$ equivalence classes] a subset $E$ is obtained for which there exist $n+1$ coordinate functions that sum to zero throughout $E$. Then the $(n+1)$ th function corresponds to the additive function $V$, the first $n$ functions to (minus) the additive value functions. This explains the symmetry of the

\footnotetext{
${ }^{3} \mathbb{R} \backslash\{0\}$ has a hole by the absence of 0 .
} 
conditions used in Theorem 2.2. By this same symmetry, Step 4.1 in the proof in Appendix A could be obtained as a corollary of Step 2.2. A similar approach can be found in Vind (1991), who characterized sets where the sum of coordinate functions is positive.

Wakker (1989b, Remark III.7.8) and the more appealing fig. 2 in Wakker (1993) showed, for three dimensions, that (3) cannot be omitted in Theorem 2.2. (All other conditions are satisfied by the interior of the domain in these examples.) Fig. 1 adapts these examples to two dimensions. The examples are also easily adapted to dimensions higher than three. By the described symmetry argument one can reformulate them to show that also (2) cannot be omitted in Theorem 2.2, not even for one coordinate (say the $n$ th): One maps, with $W$ a continuous representing function that obviously is not additive, each $x=\left(x_{1}, \ldots, x_{n-1}, x_{n}\right)$ to $\left(x_{1}, \ldots, x_{n-1}, W(x)\right)$, and orders the latter according to $x_{n}$. That way we constructed fig. 1c (thus 1b) from 1a. The necessity of condition (2) has been illustrated before by Segal (1991, Example 1); he obtained his example and conditions independently from the symmetry argument as presented here.

\section{Appendix A: Proof of Theorem 2.2}

This appendix will make use of results concerning connected order topologies. These results are presented in Appendix B.

The projections of $E$ on the coordinates are all connected (Lemma B.2). $O^{x}$ denotes the open neighborhood of $x$ where $\succcurlyeq$ is represented by the additive $V^{x}$ with additive value functions $V_{i}^{x}$. We may and will assume that all neighborhoods $O^{x}$ are cubes, so of the form $\prod_{i=1}^{n} O_{i}^{x}$, where each $O_{i}^{x}$ is of the form $] y_{i}, z_{i}[$ or $] y_{i}, \rightarrow[$ or $] \leftarrow, z_{i}\left[\right.$ or $X_{i}{ }^{4}$ We first construct the additive function $V$ without establishing that it is representing. The latter is shown subsequently.

\section{Stage 1. Choosing scale and location of the additive representation of starting cube $O^{r}$}

We take some ('reference') cube $O^{r}$ as starting domain, and define $V$ on $O^{r}$, still to be extended, as the additive representation $V^{r}$ given there. This will turn out to uniquely determine the entire function $V$.

Stage 2. Extending $V$ consistently to a cube $O^{a}$ not yet covered (see fig. 4)

\section{Step 2.1. Extending $V$ to a cube $O^{a}$ not yet covered}

\footnotetext{
${ }^{4}$ Note that, for maximal $x_{i} \in X_{i}$, this includes $\left.] y_{i}, x_{i}\right]$, as it is identical to $] y_{i}, \rightarrow[$; for minimal $x_{i} \in X_{i},\left[x_{i}, z_{i}[=] \leftarrow, z_{i}\left[\right.\right.$ is included. Also note that, by nontriviality of $\geqslant_{i}$, the form $\left[x_{i}\right]$ cannot occur. This and strong monotonicity imply that all coordinates are 'essential'.
} 


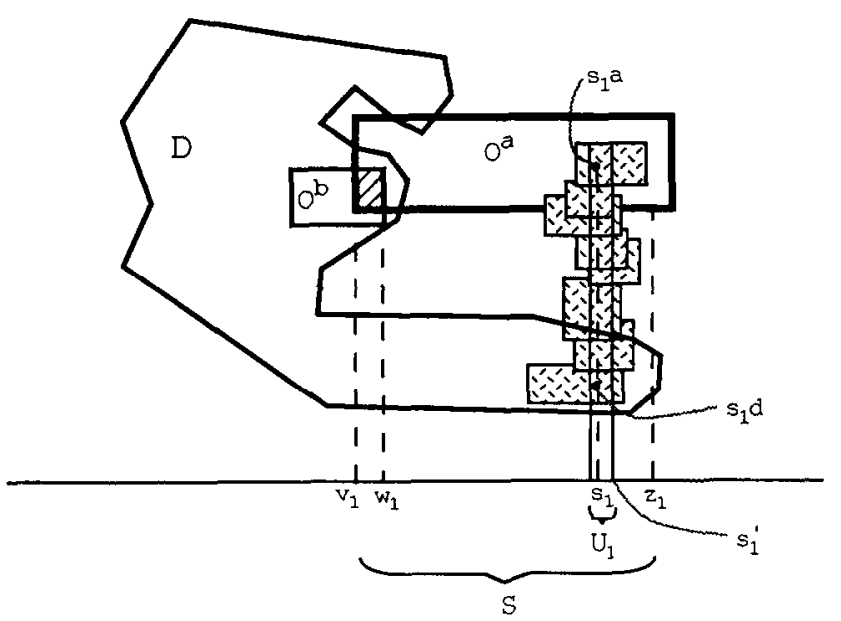

Fig. 4 (Stage 2). $\square: O^{b} \cap O^{a}, \square$ : link from $s_{1} d$ to $s_{1} a$.

Suppose we have covered a connected open domain $D \subset E$ that is a union of (possibly uncountably many) sets of the form $O^{x}$, and have rescaled all additive functions on these cubes $O^{x}$ so that they fit together as one function $V$ on $D$ that is additive.

Suppose there is a cube $O^{\alpha}$ that intersects $D$, but is not entirely contained in $D$. As pointed out in Wakker (1993), the additive functions $V^{a}$ on $O^{a}$ and $V$ on $D$ cannot immediately be fitted together on the intersection of $O^{a}$ and $D$, because this intersection does not have to be a product set, and does not have to be connected. All uniqueness results in the literature require such conditions. What we do instead is fit together $V^{a}$ with only one $(V=) V^{b}$ on the intersection of $O^{a}$ and $O^{b}$, where $O^{b}$ is one of the open cubes constituting $D$ that intersects $O^{a}$; from now on we assume that $V^{a}$ and $V^{b}$ coincide on their common domain.

The most difficult part of the proof will be the demonstration that now $V^{a}$ fits together with $V$ on the entire common domain, not just on $O^{b}$. In other words, independence from the particular choice of $O^{b}$ must be established. Actually, we must show more, we must show that $V$ can be extended to $O^{a}$ while still being additive. For the latter it is necessary (and sufficient) to show, stronger, that all separate additive value functions fit together on common domains. We show this for $V_{1}$ and $V_{1}^{a}$. KLST (subsection 6.5.5) already pointed out that consistency requirements must be fulfilled. Nevertheless this has often been overlooked in the literature.

Step 2.2. Consistency of the extension

$V_{1}$ and $V_{1}^{a}$ coincide on $O_{1}^{b} \cap O_{1}^{a}$; this contains a nonempty subset of the 
form ] $v_{1}, w_{1}$ [ (also if $O_{1}^{b} \cap O_{1}^{a}$ itself is 'unbounded'). It suffices to show that $V_{1}$ and $V_{1}^{a}$ coincide at an arbitrary $z_{1} \geqslant_{1} v_{1}$ in their common domain, the case $z_{1} \preccurlyeq_{1} w_{1}$ being similar. Obviously, the entire set $\left.\left.S:=\right] v_{1}, z_{1}\right]$ is contained in the domain of $V_{1}^{a}$. But also it is contained in the domain of $V_{1}$, i.e., $\pi_{1}(D)$, because the latter is connected and open, so we can apply Lemma B.6(c). $S$ itself is also connected by Lemma B.6(c). Let

$$
\left.\left.S^{\prime}:=\left\{s_{1} \in S: V_{1} \text { and } V_{1}^{a} \text { coincide on }\right] v_{1}, s_{1}\right]\right\} .
$$

We show that $S^{\prime}$ is open and closed within $S$, thus by connectedness of $S$ is that entire set. Let $s_{1}$ be contained in the closure of $S^{\prime}$ within $S$. We show it is contained in the interior of $S^{\prime}$ within $S$. Obviously, $s_{1} a$ is contained in the cube $O^{a}$. Take an $s_{1} d \in D$. We may assume $O^{s_{1} d} \subset D$. Since the cubes constitute an open cover of $E$, they obviously give an open cover of $\left\{x \in E: x_{1}=s_{1}\right\}$. The latter set being connected by (2), we can, by Lemma B.3, extract from the latter cover a link $O^{x^{1}}, \ldots, O^{x^{m}}$ from $s_{1} d$ to $s_{1} a$. On the open intersection $U_{1}:=O_{1}^{s_{1} d} \cap O_{1}^{x^{1}} \cap \ldots \cap O_{1}^{x^{m}} \cap O_{1}^{a}$, the respective additive value functions $V_{1}, V_{1}^{x^{1}}, \ldots, V_{1}^{x^{m}}, V_{1}^{a}$ differ only be scale and location, by standard uniqueness results applied to the intersections of respective cubes. Note that, by nontriviality of $\succcurlyeq_{i}$ and strong monotonicity, openness of these intersections implies essentially of all coordinates. Since $V_{1}$ and $V_{1}^{a}$ actually coincide on $] v_{1}, s_{1}[$, they coincide on the 'left part' of the open neighborhood $U_{1}$ of $s_{1}$, thus on the entire $U_{1}$, so in particular on $\left.] v_{1}, s_{1}^{\prime}\right]$ for an $s_{1}^{\prime} \succ_{1} s_{1}$ (or $s_{1}^{\prime}=s_{1}$ if $s_{1}$ is maximal in $X_{1}$ ). So $s_{1}$, if contained in the closure of $S^{\prime}$, is actually contained in the interior of $S^{\prime}$ within $S$, and the nonempty $S^{\prime}$ is open and closed within the connected $S$. It must be identical to the latter. Indeed $V_{1}$ and $V_{1}^{a}$ coincide at $z_{1} \in S$. Since $z_{1}$ was chosen arbitrarily, $V_{1}$ and $V_{a}^{1}$ coincide on their entire domain. We conclude that $V$ can be extended additively to $O^{a}$. Note that by Lemma B.1 the new domain is again connected.

\section{Stage 3. Covering the entire domain $E$}

Intuitively, the additive function can be extended as long as there are open $O^{a}$ on which $V$ has not yet been defined, and that intersect the domain already covered. In general, existence of a maximal domain is guaranteed by the Lemma of Zorn from the set-theoretic axiomatics. Elaboration is omitted. By connectedness of $E$, and Lemma B.3, this process will only stop if the entire domain $E$ has been covered.

\section{Stage 4. The additive function is representing}

Step 4.1. If an additive function $V$ is locally representing, then it is constant on equivalence classes 
Consider an equivalence class $[x]$. Let the set $S \subset[x]$ be the set of alternatives that have the same $V$-value as $x$, and take $y$ in the closure of $S$ within [x]. Within $O^{y}$, the open neighborhood of $y$ on which $V$ is representing, $V$ is constant on $[x]$. Because $y$ is in the closure of $S$, there is an element of $S$ in $O^{y} ; V$, constant on $[x] \cap O^{y}$, must be $V(x)$ there. So, as soon as $y$ is contained in the closure of $S$, there is actually an open neighborhood $[x] \cap O^{y}$ relative to $[x]$ of $y$ that is entirely contained in $S$. Apparently, the nonempty $S$ is both open and closed within $[x]$ : it must be the entire $[x]$. So $V$ is constant on equivalence classes.

\section{Step 4.2. $V$ is globally representing}

In view of Step 4.1, it suffices to show for any $x$ that on $S:=] x, \rightarrow[, V$ is strictly larger than $V(x) . S^{\prime}=\{y \in S$ : for all $z$ in $\left.\left.] x, y\right]: V(z)>V(x)\right\}$. Let $s$ be in the closure of $S^{\prime}$, relative to $] x, \rightarrow\left[\right.$. On $O^{s}, V$ is representing. Since $s$ is in the closure of $S^{\prime}, O^{s}$ also contains an element $s^{\prime}$ of $S^{\prime}$. We take $s^{\prime}=s$ (so $s^{\prime} \leqslant s$ ) if $s$ itself is contained in $S^{\prime}$, otherwise one can derive $s^{\prime} \leqslant s$ from the form of $S^{\prime}$. The open $O^{s}$ contains an alternative $t>s$ (unless $s$ is maximal in the open $E$, which may happen if the $X_{i}$ 's have maximal coordinates; then take $t=s$ ). By Lemma B.6(c), $O^{s}$ is connected, and contains an element of every equivalence class between $s^{\prime}$ and $t$. Apparently, on all these equivalence classes $V$ has a value at least as large as $V\left(s^{\prime}\right)$, so strictly greater than $V(x)$. So as soon as an alternative $s$ is contained in the closure of $S^{\prime}$, there exists an open neighborhood $] x, t[(] x, s]$ if $s$ is maximal) entirely within $S^{\prime} . S^{\prime}$ must be open and closed, from $O^{x}$ we see that it is nonempty, so $S^{\prime}$ is identical to $S$, the latter being connected by Lemma B.6(c). Indeed $V$ is strictly larger than $V(x)$ on $] x, \rightarrow[$.

We also show, briefly:

Step 4.3. Each $V_{j}$ represents $\succcurlyeq_{j}$ on $\pi_{j}(E)$

This follows since $V_{j}$ represents $\geqslant_{j}$ locally, $X_{j}$ [thus $\left.\pi_{j}(E)\right]$ is endowed with the $\succcurlyeq_{j}$ order topology, and $\pi_{j}(E)$ is connected (use Lemma B.3).

\section{Stage 5. Continuity and uniqueness}

By Theorem 3.1 in Wakker (1988), the local additive representations are continuous, so that the ranges of the local $V_{j}^{x}$ 's are connected. Continuity of all $V_{j}$ 's (thus of $V$ ) now follows since their ranges are a union of nondisjoint intervals, so are connected and contain no gaps. Cardinality follows from cardinality of $V^{r}$ on the starting domain $O^{r}$, and the uniqueness of the extension of $V$ from there on. 


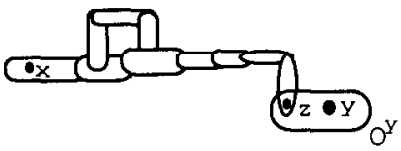

Fig. 5. (Proof of Lemma B.3). Assume connectedness. Then all points are linked to $x$ : Let $y$ be in the closure of $L$, the set of points linked to $x$. For $y \in O^{y} \in O, O^{y}$ contains a $z \in L$. $O^{y}$ links $y$, as well as any other of its elements, to $z$, thus to $x$. So $L$ is both open and closed. By connectedness, $L=Y$. Note that the roundabout part of the link (to the right of $x$ ) can be omitted.

\section{Appendix B: Elementary topological results}

This appendix lists some elementary properties of connected topologies. The first two are well-known, so are given without proof.

Lemma B.I. If two connected subsets intersect, their union is again connected.

Lemma B.2. If $f$ is a continuous mapping and $Y$ is connected then $f(Y)$ is connected as well.

The lemma below gives a characterization of connected topological spaces; it may serve to motivate the term connected.

Lemma B.3. Let $Y$ be a connected topological space. Let $O$ be an open covering. Then every pair of elements $x, y$ of $Y$ is linked, meaning there exists a link, i.e., finitely many open $O_{1}, \ldots, O_{m} \in O$ such that $x \in O_{1}, y \in O_{m}$, and each subsequent pair of $O_{j}$ 's intersects. Further $O_{i} \cap O_{i-k}$ can be taken empty for every $k \geqq 2$. Conversely, for a topological space, if for every open covering each pair of elements is linked, then the space is connected.

Proof. See fig. 5. (The reversed implication is an immediate consequence of the definition of connectedness.)

The proof of the following elementary result is omitted. The result has been used several times without explicit mention.

Lemma B.4. Suppose that $\succcurlyeq$ is a weak order that is continuous with respect to a connected topology. Let $x>z$. Then there exists a y such that $x>y>z$.

The following result is given as Proposition 1 on p. 81 in Bourbaki (1971, Chapter I). 
Lemma B.5. Let $D$ be a connected set. Then so is its closure $\operatorname{cl}(D)$, and, more generally, any set $D^{\prime}$ with $D \subset D^{\prime} \subset \operatorname{cl}(D)$.

Lemma B.6. Let $Y$ be endowed with a weak order $\geqslant$ and the order topology; suppose $Y$ is connected. Then:

(a) Sets $[x, y]$ are compact.

(b) $S \subset Y$ is connected if and only if, for every $x<z \in S$, and $x \preccurlyeq y \leqslant z$, there exists a $y^{\prime} \in S$ with $y^{\prime} \sim y$.

(c) If $S \subset Y$ is open or closed, then it is connected if and only if it is a preference interval, i.e., a set of the form *, \#, where for * one either substitutes $] \leftarrow$, or $] x$, or $[x$, and for \# either $\rightarrow[$, or $y[$, or $y]$ (note that $Y$ can be denoted as $] \leftarrow, \rightarrow[$ ).

Proof. Part (a) is obtained from Lemma B.3 by linking endpoints; (b) and (c) are derived below. Assume, for nontriviality, that $\varnothing \neq S \neq Y$, and that $S$ contains two nonequivalent elements $a \prec b$.

First suppose that $S$ is connected, and let $x \prec z$ be elements of $S$. Suppose, for $x \prec y \prec z$, that $S$ contains no element equivalent to $y$. Then the sets (]$\leftarrow, y[) \cap S$ and (]$y, \rightarrow[) \cap S$ give a violation of connectedness of $S$.

Next we show that the condition described in (b) above implies connectedness of $S$. Define $[S]:=\{y \in Y: y \sim s$ for an $s \in S\}$, and define $S^{-}:=\{x \in Y: x \leqslant s$ for all $s \in[S]\} . S^{-}$is an intersection of closed sets, so is closed again.

Suppose that $S^{-}$is nonempty. $S^{-} \neq Y$ because $b \notin S^{-}$. So, by connectedness of $Y, S^{-}$cannot be open, and $S^{-} \neq \bigcup_{s \in S^{-}}\{y \in Y: y<s\}$. There must exist an element $s^{-}$of $S^{-}$that is best, i.e., $s^{-} \geqslant s$ for all $s \in S^{-}$. It follows straightforwardly that $[S]$ contains $\left.] s^{-}, x\right]$ for each $x \in[S]$.

If $S^{-}$is empty, then from the condition in (b) it follows that [S] contains ]$\leftarrow, x]$ for each $x \in[S]$.

Define $S^{+}:=\{x \in Y: x \geqslant s$ for all $s \in[S]$ ). It is demonstrated similarly that either $S^{+}$is nonempty, in which case it contains a worst element $s^{+}$and $[S]$ contains $\left[x, s^{+}\left[\right.\right.$for each $x \in[S]$, or $S^{+}$is empty, in which case $[S]$ contains $[x, \rightarrow[$ for each $x \in[S]$.

By distinguishing cases ${ }^{5}$ it now follows straightforwardly that $[S]$ is a preference interval. It is well-known that such sets are connected; see for instance Lemma VI.7.4 in Wakker (1989b). This implies connectedness of $S$.

As openness or closedness of $S$ implies that $S=[S]$, the above reasoning also gives (c).

\section{Appendix C: Applications to rank-ordered sets and other cases}

We next present some special cases of the results obtained above. The

\footnotetext{
${ }^{5}$ In the notation of (c), for $[S]$ instead of $\left.S,{ }^{*}=\right] \leftarrow$ if $S^{-}$is empty, $\left.\left.{ }^{*}=\right] x=\right] s^{-}$if $s^{-} \notin[S]$ and the latter is not empty, ${ }^{*}=\left[x=\left[s^{-}\right.\right.$if $s^{-} \in[S]$, similarly for \# and $S^{+}$and $s^{+}$.
} 
following lemma shows that on full product sets all connectedness requirements of this paper are satisfied. The only nontrivial condition is connectedness of equivalence classes.

Lemma C.I Suppose that $\geqslant$ is a weak order on $\prod_{i=1}^{n} X_{i}$, monotonic with respect to binary relations $\vartheta_{i}$ on $X_{i}$ that generate a connected order topology, and continuous with respect to the product topology. Then its equivalence classes are connected.

Proof. We first consider some special cases.

Case 1. Suppose that each $\succcurlyeq_{i}$ is an order, i.e., equivalent coordinates are identical. For connectedness of an equivalence class $[x]$, it suffices to show that each pair of elements $y \neq z$ is contained in a connected subset $S \subset[x]$.

Case 1.1. Let $y_{1} \succ_{1} z_{1}, y_{2} \prec_{2} z_{2}, y_{3}=z_{3}, \ldots, y_{n}=z_{n}$. We define $S:=[x] \cap$ $\left(\left[z_{1}, y_{1}\right] \times\left[y_{2}, z_{2}\right] \times\left\{y_{3}\right\} \times \ldots \times\left\{y_{n}\right\}\right)$, and show $S$ is connected. Consider the restriction of $\pi_{1}$ to $S$; it is continuous. To see that $\pi_{1}(S)=\left[z_{1}, y_{1}\right]$ we suppose $v_{1} \in\left[z_{1}, y_{1}\right]$, and show there exists $v_{2} \in\left[y_{2}, z_{2}\right]$ such that $\left(v_{1}, v_{2}, z_{3}, \ldots, z_{n}\right) \sim x$ : The sets $\left\{w_{2} \in\left[y_{2}, z_{2}\right]: \quad\left(v_{1}, w_{2}, y_{3}, \ldots, y_{n}\right) \geqslant x\right\}$ and $\left\{w_{2} \in\left[y_{2}, z_{2}\right]\right.$ : $\left.\left(v_{1}, w_{2}, y_{3}, \ldots, y_{n}\right) \leqslant x\right\}$ are closed [this is straightforward from continuity of $\geqslant$; elaboration is given in Lemma 0.2.1 of Wakker (1989b)], they are nonempty for containing $z_{2}$ and $y_{2}$ respectively, and their union is $\left[y_{2}, z_{2}\right]$, so by connectedness of the latter they must intersect; $v_{2}$ is taken from that intersection.

From monotonicity and the assumption that $\succcurlyeq_{2}$ is an order it follows that $v_{2}$, thus $\left(v_{1}, v_{2}, z_{3}, \ldots, z_{n}\right)$, is uniquely determined. So $\pi_{1}$ is a bijection from $S$ to $\left[z_{1}, y_{1}\right]$. Continuity of $\pi_{1}$, and compactness of its domain $S$ (being a closed subset of the compact $\left[z_{1}, y_{1}\right] \times\left[y_{2}, z_{2}\right] \times\left[y_{3}\right] \times \ldots \times\left[y_{n}\right]$, by Lemma B.6(a) and the fact that a product of compact spaces is again compact), implies that also $\pi_{1}^{-1}$ is continuous. $S$ is the continuous image of a connected domain, so is again connected.

Case 1.2. Here the remainder of Case 1 is considered, i.e., now $y$ and $z$ are general. $S$ will be a union of sets $S^{1}, \ldots, S^{k}$, constructed by means of alternatives $y=y^{0}, y^{1}, \ldots, y^{k}=z$. There exists a coordinate $i$ such that $y_{i} \succ_{i} z_{i}$ and a coordinate $j$ such that $y_{j}<_{j} z_{j}$.

If $z_{i}\left(z_{j} y\right) \preccurlyeq x$, then, analogously to the reasoning in the beginning of Case 1.1 , there exists $z_{i}^{\prime}$ between $z_{i}$ and $y_{i}$ such that $z_{i}^{\prime}\left(z_{j} y\right) \sim x$, and $y^{1}:=z_{i}^{\prime}\left(z_{j} y\right)$ is defined.

If $z_{i}\left(z_{j} y\right) \geqslant x, z_{j}^{\prime}$ between $y_{j}$ and $z_{j}$ is found to give $z_{i}\left(z_{j}^{\prime} y\right) \sim x$, and $y^{1}:=z_{i}\left(z_{j}^{\prime} y\right)$ is defined.

In any case, we have found $y^{1} \sim x$, differing from $y$ by only two 
coordinates, and with one more coordinate identical to the coordinates of $z$, than $y$. We can define a connected set $S^{1} \subset[x]$ containing the pair $y, y^{1}$ as constructed in Case 1.1. Next we construct, similarly, an alternative $y^{2}$ from $y^{1}$ that differs from $y^{1}$ by only two coordinates, and that has at least one coordinate more identical with $z$, than $y^{1}$, and subsequently we obtain again a connected set $S^{2} \subset[x]$ containing both $y^{1}$ and $y^{2}$. We continue this way, after at most $n$ steps (actually $n-1$ ) we obtain $y^{k}=z$.

All sets $S^{1}, S^{2}, \ldots$ as constructed above are connected, each subsequent pair intersects, so their union $S$ is again connected.

Case 2. The general case, where not all $\geqslant_{i}$ relations are orders, now follows by means of the map $\left(x_{1}, \ldots, x_{n}\right) \mapsto\left(\left[x_{1}\right], \ldots,\left[x_{n}\right]\right)$. The image space is endowed with the naturally generated structures. Connectedness of equivalence classes in the image space, as established above, then implies the same in the original space; note here that in the original space equivalent coordinates are not 'separated' topologically.

Next we turn to 'rank-ordered' sets. We use below the following structural assumption.

Assumption C.2 (a). E is a rank-ordered set, i.e., there is given a weak order $\succcurlyeq^{\prime}$ on a nonempty set $X$, and $E:=\left\{\left(x_{1}, \ldots, x_{n}\right) \in X^{n}: x_{1} \geqslant^{\prime} \ldots \geqslant^{\prime} x_{n}\right\}$. The order topology generated by $\succcurlyeq$ is connected.

(b) The binary relation $\succcurlyeq$ on $E$ is a weak order, monotonic with respect to $\succcurlyeq^{\prime}$, and continuous with respect to the product topology. Further $n \geqq 2$.

Lemma C.3. Under Assumption C.2, the equivalence classes of $\geqslant$ are connected. So are $E$, and each set of the form $\left\{x \in E: x_{i}=s_{i}\right\}$ for some $i, s_{i}$. Also the intersections of these sets with int $(E)$ are connected.

Proof. The proof that equivalence classes are connected is analogous to that of Lemma C.1. Only one adaptation is needed. In Case 1.2 there, $z_{i}\left(z_{j} y\right)$ may not be contained in $E$. It may happen that $z_{i} \prec^{\prime} y_{i+1}$, or $z_{j} \succ^{\prime} y_{j-1}$. This is avoided by choosing $i$ as the largest coordinate for which $y_{i} \succ^{\prime} z_{i}$, and $j$ as the smallest coordinate for which $y_{j} \prec^{\prime} z_{j}$. Then, if $i \leqq n-1$, $y_{i} \succ^{\prime} z_{i} \succcurlyeq^{\prime} z_{i+1} \succcurlyeq^{\prime} y_{i+1}$, and if $j \geqq 2, y_{j} \prec^{\prime} z_{j} \preccurlyeq^{\prime} z_{j-1} \preccurlyeq^{\prime} y_{j-1}$. Also the proof that intersections of equivalence classes with int $(E)$ are connected, is analogous. The derivation of Case 1.1. in Lemma C.1 does not need modification, primarily because the set $S$ is contained in int $(E)$ if $y$ and $z$ are. The derivation of Case 1.2 does not need further modification, other than described above, and neither does the derivation of Case 2 there.

Next we prove that $E$ itself is connected. Let $y, z \in E$. We construct a connected set $S=S^{1} \cup \ldots \cup S^{k}$ within $E$ that contains both $y$ and $z$. Define 
$y^{0}:=y$. Let $i$ be the largest coordinate for which $y_{i} \geqslant^{\prime} z_{i}, y_{i} \neq z_{i}$. The set $S^{1}:=\left\{v_{i} y: y_{i} \succcurlyeq^{\prime} v_{i} \succcurlyeq^{\prime} z_{i}\right\}$ is entirely contained in $E$ (since $z_{i} \geqslant^{\prime} z_{i+1} \geqslant^{\prime} y_{i+1}$ if $i \leqq n-1)$ and is homeomorphous with $\left[z_{i}, y_{i}\right]$, thus is connected. Define $y^{1}:=z_{i} y$; it has one more coordinate in common with $z$ than $y$. We continue inductively, until a $y^{k^{\prime}}$ has been obtained for which no coordinate is weakly preferred to, but different from, coordinates of $z$. Then we take the smallest coordinate $j$ for which $y_{j}^{k^{\prime}} \preccurlyeq^{\prime} z_{j}$ but $y_{j}^{k^{\prime}} \neq z_{j}$ and we construct $y^{k^{\prime}+1}$ and $S^{k^{\prime}+1}$ in the same way as we constructed $S^{1}$. Since each time one more coordinate becomes identical to one of $z$, the process will stop with $y^{k}=z$. Each subsequent pair $S^{m}, S^{m+1}$ intersects, hence their union can be defined as the connected set $S$ containing $y$ and $z$. The demonstration that $\operatorname{int}(E)$ is connected is similar, if $y$ and $z$ are contained in int $(E)$ then so are $S^{1}, \ldots, S^{k}$, and $y^{1}, \ldots, y^{k}$.

The reasoning used to prove connectedness of $E$, can also be used to prove connectedness of sets $\left\{x \in E: x_{i}=s_{i}\right\}$, as well as thcir interscctions with int $(E)$. If $y_{i}=z_{i}=s_{i}$ then the set $S$ constructed above is entirely contained in $\left\{x \in E: x_{i}=s_{i}\right\}$.

Lemma C.4. Under Assumption C.2, all alternatives other than the extreme ones are matched.

Proof. Note that, by rank-orderedness, an alternative $\left(x_{1}, \ldots, x_{n}\right)$ always has, for some $0 \leqq k \leqq l \leqq n$, its coordinates $x_{1}, \ldots, x_{k}$ maximal, $x_{k+1}, \ldots, x_{l}$ neither maximal nor minimal, and $x_{l+1}, \ldots, x_{n}$ minimal.

Suppose that $\mu \in X$ is a maximal outcome with respect to $\geqslant$, and that all coordinates of $x \in E$ are neither maximal nor minimal. Then $\left(\mu, x_{2}, \ldots, x_{n}\right)$ is interior-matched, because by continuity and connectedness it is equivalent to some $\left(\mu^{\prime}, x_{2}^{\prime}, x_{3}, \ldots, x_{n}\right) \in \operatorname{int}(E)$ with $\mu \succ^{\prime} \mu^{\prime} \succ^{\prime} x_{2}^{\prime} \succ^{\prime} x_{2}$. Inductively, $\left(\mu, \ldots, \mu, x_{k+1}, \ldots, x_{n}\right)$ is $k$ th order matched for each $k \leqq n-1$, being equivalent to some $(k-1)$ th order matched alternative $\left(\mu, \ldots, \mu, \mu^{\prime \prime}, x_{k+1}^{\prime}, \ldots, x_{n}\right)$ with $\mu \succ^{\prime} \mu^{\prime \prime} \succ^{\prime} x_{k+1}^{\prime} \succ^{\prime} x_{k+1}$. Similarly, if $v \in X$ is minimal with respect to $\succcurlyeq^{\prime}$, then, with all coordinates of $x \in E$ neither maximal nor minimal, $\left(x_{1}, \ldots, x_{n-k}, v, \ldots, v\right)$ is $k$ th order matched for each $k \leqq n-1$.

So maximal $x_{1}, \ldots, x_{n-1}$ and minimal $x_{2}, \ldots, x_{n}$ occur in matched alternatives. It shows that all non-extreme alternatives are matched.

From the above results, the inclusions int $(E) \subset E \subset \operatorname{cl}(\operatorname{int}(E))$ for rankordered sets $E$, and Theorem 3.3, we conclude:

Corollary C.5. Under Assumption C.2, additivity axioms for $\geqslant$ imply the existence of continuous additive value functions for $\succcurlyeq$ on E\\{extreme alternatives\}. 
Next we turn to the product of rank-ordered sets. These are important in cumulative prospect theory, the new version of prospect theory developed in Tversky and Kahneman (1992) and Wakker and Tversky (1991). Besides rank-dependence also 'sign-dependence' is important here, and additivity axioms hold on subsets that are products of two rank-ordered subsets. A typical example of such a set is $\left\{\left(x_{1}, \ldots, x_{5}\right) \in \mathbb{R}^{5}: x_{1} \geqq x_{2} \geqq x_{3} \geqq 0 \geqq x_{4} \geqq x_{5}\right\}$, a product of a rank-ordered subset of $\mathbb{R}_{+}^{3}$ and one of $\mathbb{R}_{-}^{2}$. Its elements do not only induce the same ordering of coordinates $\left(x_{1} \geqq \ldots \geqq x_{5}\right)$, but also have the same 'sign profile'. Note that $E$ below is at least two-dimensional. No further restriction is imposed on the dimension of $E$.

Theorem C.6. Let $\succcurlyeq$ be a weak order on $E^{1} \times E^{2}$, a product of two rank-ordered sets, each of which satisfies Assumption C.2(a) with nontrivial $\geqslant 1^{\prime}$ and $\geqslant^{2}$, Suppose $\succcurlyeq$ is a weak order on $E^{1} \times E^{2}$ that satisfies monotonicity with respect to $\succcurlyeq^{\prime}$ and $\succcurlyeq^{2}$, is continuous with respect to the product topology, and satisfies the additivity axioms. Then there exists a continuous real-valued additive representation for $\geqslant$ that is cardinal.

Proof. On int $(E)$ all conditions of the Structural Assumption 2.1 are satisfied. The derivation of this is similar to the derivation on rank-ordered sets, and will not be repeated. Hence there exists, by Theorem 2.3, an additive representation on int $(E)$. For the extension to boundary alternatives, note that int $(E) \subset E \subset \operatorname{cl}($ int $(E))$ is direct. By Corollary 3.3 it suffices to show that all alternatives are matched.

To this end, take any interior $r^{1} \in E^{1}$ and interior $r^{2} \in E^{2}$, with nonextreme coordinates. By $\left(\left(r_{1}^{1}, r_{2}^{1}, r_{3}^{1}, \ldots\right),\left(r_{1}^{2}, r_{2}^{2}, r_{3}^{2}, \ldots\right)\right)$, or $\left(r^{1}, r^{2}\right)$, we denote the related element of $E$. Let $\mu$ be a maximal outcome related to the rank-ordered set $E^{1}$. To see that there exists a matched alternative with $\mu$ as $k$ th coordinate, for each $k$, note, by continuity and connectedness, that there exist nonextreme outcomes $\tau<\mu$ and $\tau^{\prime}<r_{1}^{2}$ such that

$$
\left(\left(\mu, \ldots, \mu, r_{k+1}^{1}, \ldots\right),\left(\tau^{\prime}, r_{2}^{2}, r_{3}^{2}, \ldots\right)\right) \sim\left(\left(\tau, \ldots, \tau, r_{k+1}^{1}, \ldots\right),\left(r_{1}^{2}, r_{2}^{2}, r_{3}^{2}, \ldots\right)\right) .
$$

First, for $k=1$, this shows that there exists an interior-matched alternative with $\mu$ as first coordinate. Next, inductively, it shows that for each $k$ there exists a $k$ th order matched alternative with $\mu$ as $k$ th coordinate.

A similar rcasoning shows that, in relation to $E^{2}$, maximal outcomes also occur as $k$ th coordinate for matched alternatives within $E^{2}$, for each $k$. Similar observations hold as well for minimal outcomes. This proves that all alternatives are matched.

Finally, we add a topological observation. 
Remark C.7. Suppose we modify Assumption C.2(a) by replacing the topology on $X$ generated by $\succcurlyeq^{\prime}$ by any connected topology. Suppose we then require continuity of $\succcurlyeq^{\prime}$ with respect to this topology, and continuity of $\geqslant$ with respect to the product topology of the new topology. Then Corollary C.5 and Theorem C.6 remain valid.

Proof. We derive continuity of $\succcurlyeq$ with respect to the product of order topologies. Say $x>y$. Take, by connectedness and monotonicity [similarly to Wakker (1989, proof of Lemma VI.7.6)], $\tilde{x}_{n}$ such that either $\tilde{x}_{n}=x_{n}$ if $x_{n}$ is minimal, or $\tilde{x}_{n} \prec x_{n}$ (we write $\prec$ for $\prec^{\prime}$ or $<^{1{ }^{\prime}}$ or $\prec^{2}$ ) and still $\tilde{x}_{n} x>y$. Next take $\tilde{x}_{n-1}$ such that either $\tilde{x}_{n-1}=x_{n-1}$ if $x_{n-1}$ is minimal, or $\tilde{x}_{n} \leqslant \tilde{x}_{n-1} \prec x_{n-1}$ and still $\tilde{x}_{n-1}\left(\tilde{x}_{n} x\right) \succ y$; etc. One thus ends up with a $\tilde{x} \succ y$, and through $\tilde{x}$ constructs a set of alternatives strictly preferred to $y$, open with respect to the product of order topologies, and containing $x$; etc.

\section{References}

Blaschke, W. and G. Bol, 1938, Geometrie der Gewebe (Springer, Berlin).

Bourbaki, N., 1971, Eléments de mathématiques, topologie générale (Chapitres 1 à 4) (Diffusion CCLS, Paris).

Chew, S.H. and L.G. Epstein, 1989a, Axiomatic rank-dependent means, Annals of Operations Research 19, 299-309.

Chew, S.H. and L.G. Epstein, 1989b, A unifiying approach to axiomatic non-expected utility theories, Journal of Economic Theory 49, 207-240.

Debreu, G., 1960, Topological methods in cardinal utility theory, in: K.J. Arrow, S. Karlin and P. Suppes, eds., Mathematical methods in the social sciences (Stanford University Press, Stanford, CA) 16-26.

Debreu, G., 1964, Continuity properties of Paretian utility, International Economic Review 5 , 285-293.

Fishburn, P.C., 1976, Utility independence on subsets of product sets, Operations Research 24, $245-255$.

Fishburn, P.C., 1988, Nonlinear preference and utility theory (Johns Hopkins University Press, Baltimore, MD).

Fishburn, P.C., 1992, A general axiomatization of additive measurement with applications, Naval Research Logistics 39, 741-755.

Gorman, W.M., 1968, The structure of utility functions, Review of Economic Studies 35, $367-390$.

Green, J. and B. Jullien, 1988, Ordinal independence in non-linear utility theory, Journal of Risk and Uncertainty 1, 355-387 (Erratum, 2, 1989, 119.)

Jaffray, J.Y., 1974a, Existence, propriétés de continuité, additivité de fonctions d'utilité sur un espace partiellement ou totalement ordonné, Ph.D. dissertation (Université de Paris VI, Paris).

Jaffray, J.Y., 1974b, On the extension of additive utilities to infinite sets, Journal of Mathematical Psychology 11, 431-452.

Krantz, D.H., R.D. Luce, P. Suppes and A. Tversky (KLST), 1971, Foundations of Measurement 1 (Additive and Polynomial Representations) (Academic Press, New York).

Quiggin, J., 1982, A theory of anticipated utility, Journal of Economic Behavior and Organization 3, 323-343.

Schmeidler, D., 1989, Subjective probability and expected utility without additivity, Econometrica 57, 571-587.

Segal, U., 1991, Additively separable representations on non-convex sets, Journal of Economic Theory 56, 89-99. 
Tversky, A. and D. Kahneman, 1992, Advances in prospect theory: Cumulative representation of uncertainty, Journal of Risk and Uncertainty 5, 297323.

Vind, K., 1987, Additive utility functions and other special functions in economic theory, Lecture Notes (Institute of Economics, University of Copenhagen, Copenhagen).

Vind, K., 1991, Independent preferences, Journal of Mathematical Economics 20, 119-135.

Wakker, P.P., 1986, Representations of choice situations, Ph.D. dissertation (University of Brabant, Tilburg, The Netherlands).

Wakker, P.P., 1988, The algebraic versus the topological approach to additive representations, Journal of Mathematical Psychology 32, 421-435.

Wakker, P.P., 1989a, Continuous subjective expected utility with nonadditive probabilities, Journal of Mathematical Economics 18, 1-27.

Wakker, P.P., 1989b, Additive representations of preferences, A new foundation of decision analysis (Kluwer Academic Publishers, Dordrecht).

Wakker, P.P., 1990, From local to global additive representation (Duke University, Fuqua School of Business, Durham, NC).

Wakker, P.P., 1991, Additive representations on rank-ordered sets I: The algebraic approach, Journal of Mathematical Psychology 35, 501-531.

Wakker, P.P., 1993, Additive representations on rank-ordered sets II: The topological approach, Journal of Mathematical Economics 22, 1-26.

Wakker, P.P. and A. Tversky, 1991, An axiomatization of cumulative prospect theory, Journal of Risk and Uncertainty, forthcoming. 